\title{
New Application of Real-time Cell-monitoring Analysis System to Detect Responses of Human Umbilical Vein Endothelial Cells to Paclitaxel
}

\author{
Mai Hazekawa," Takuya Nishinakagawa, \\ Tomoyo Kawakubo-Yasukochi, and Manabu Nakashima \\ Department of Immunological and Molecular Pharmacology, Faculty of Pharmaceutical Science, \\ Fukuoka University, 8-19-1 Nanakuma Jonan-ku, Fukuoka 814-0180, Japan
}

(Received January 30, 2020; accepted May 14, 2020)

Keywords: angialgia, phlebitis, RTCA system, side effect, paclitaxel

Although widely used in the treatment of common cancers, paclitaxel causes angialgia/ phlebitis in $60-70 \%$ of patients when administered intravenously to the upper extremities. However, useful laboratory models for the study of angialgia and phlebitis are currently lacking. The purpose of this study was to establish a new application of the real-time cellmonitoring analysis (RTCA) system to detect responses of human umbilical vein endothelial cells (HUVECs) to paclitaxel. We examined the possibility of applying this method to detect the risk of developing angialgia/phlebitis as a side effect of anticancer reagents. HUVECs were seeded onto an E-plate. After $24 \mathrm{~h}$ of culture, paclitaxel and carboplatin were added to each well and changes in electrical impedance were then recorded for $72 \mathrm{~h}$. Electrical impedance, outputted as cell index (CI) values, enabled the time-dependent monitoring of the effects of chemotherapeutics on HUVEC viability. Paclitaxel caused a greater concentration- and timedependent decrease in HUVEC CI values than with carboplatin. These findings agree with clinical case data showing that taxanes cause angialgia/phlebitis more frequently than platinum therapies. Furthermore, CI values correlated with increased levels of the inflammatory markers intercellular adhesion molecule-1 (ICAM-1), vascular cell adhesion molecule-1 (VCAM-1), and phospho-nuclear factor (P-NF)- $\mathrm{B}$. Together, these data suggest that our RTCA-based experimental method provides a useful in vitro model for the study of chemotherapy-induced angialgia/phlebitis.

\section{Introduction}

The real-time cell-monitoring analysis (RTCA) system was previously developed for the continuous monitoring of adherent cell cultures. ${ }^{(1)}$ This label-free and noninvasive method is based on the measurement of electrical impedance between interdigitated regions on the base of tissue culture plates. The impedance measurement provides quantitative information about the biological status of adherent cells, enabling the real-time monitoring of cell number, viability, and morphology. ${ }^{(2-4)}$

\footnotetext{
*Corresponding author: e-mail: mhaze@fukuoka-u.ac.jp
} https://doi.org/10.18494/SAM.2020.2818 
In previous studies, we have assessed the cytotoxicity of imatinib ${ }^{(5,6)}$ and other anticancer reagents $^{(7)}$ for oral squamous cell carcinoma using an RTCA system for real-time measurements and the WST-8 (5-[2,4-bis(sodiooxysulfonyl)phenyl]-3-(2-methoxy-4-nitrophenyl)-2-(4nitrophenyl)-2H-tetrazole-3-ium) assay for end-point measurements. Although the principle of the RTCA system is not a direct cytotoxicity evaluation, it was found that there is a positive correlation between the cell index (CI) value and the viable cell number evaluated using WST- 8 assay.

Data from our previous studies also revealed that $\mathrm{IC}_{50}$ values obtained with the RTCA system were lower than those obtained using the WST-8 assay, suggesting that the RTCA system provides a more sensitive method of evaluating cytotoxicity, enabling cellular responses to be monitored immediately after the time of treatment. ${ }^{(7)}$ Given our previous findings, we postulated that the RTCA system may also provide a powerful method of detecting the adverse effects of anticancer drugs on normal cells, such as those of the vasculature.

Paclitaxel is a microtubule-stabilizing agent that is commonly used in the treatment of cancer. It is used in combination with carboplatin (so-called TC therapy) as a standard chemotherapy for ovarian and breast cancer. Angialgia/phlebitis is an atypical adverse drug reaction associated with TC therapy. ${ }^{(8)}$ If the development of severe angialgia/phlebitis can be prevented, patients are often able to continue effective chemotherapy without a decrease in quality of life (QOL). However, the mechanism by which intravenously administered paclitaxel induces angialgia/phlebitis in patients remains unclear. Furthermore, useful experimental models for the evaluation of angialgia/phlebitis caused by paclitaxel are currently lacking.

Here, we evaluated the applicability of a new RTCA device in the study of paclitaxelassociated angialgia/phlebitis using human umbilical vein endothelial cells (HUVECs) as a model system of the human vascular endothelium. This experimental system provides useful insights into the time-dependent cellular responses that may occur in the vascular endothelium in response to the intravenous administration of chemotherapeutics, using paclitaxel as an example. Such a system may have utility in the identification and development of markers for predicting angialgia/phlebitis caused by paclitaxel and for developing methods for minimizing adverse drug responses associated with this and other anti-cancer agents.

\section{Materials and Methods}

\subsection{Reagents}

Paclitaxel and carboplatin were purchased from Wako Pure Chemical Industries, Ltd. (Osaka, Japan) and stored at $-20{ }^{\circ} \mathrm{C}$. Paclitaxel was diluted to $2 \mathrm{mM}$ in $75 \%$ ethanol and carboplatin was diluted to $25 \mathrm{mM}$ in distilled water.

\subsection{Cell culture}

HUVECs were purchased from PromoCell (Heidelberg, Germany). The cells were cultured in endothelial cell basal medium (MV-2 kit; Takara Bio Inc., Shiga, Japan) including supplements and 10\% fetal calf serum (Biowest, Nuaille, France). Cell cultures were maintained at $37{ }^{\circ} \mathrm{C}$ in cell culture plates in a humidified atmosphere of $95 \%$ air and $5 \% \mathrm{CO}_{2}$. 


\subsection{Measurement of HUVEC proliferation by RTCA cytotoxicity assay}

Real-time CI data were acquired using the iCELLigence RTCA system (ACEA Biosciences, Inc., San Diego, CA, USA). Monitoring for all treatments was performed at $37^{\circ} \mathrm{C}$ in a regulated atmosphere containing $5 \% \mathrm{CO}_{2}$.

\subsubsection{Single-agent treatment with paclitaxel or carboplatin}

E-plates (culture plate for the iCELLigence system) containing $200 \mu \mathrm{l}$ of culture medium per well were equilibrated to $37{ }^{\circ} \mathrm{C}$, and the electrical microimpedance signal, which corresponds to the $\mathrm{CI}$ value, was set to zero to establish baseline conditions. Cells $\left(1 \times 10^{4}\right.$ cells/well, unless specified otherwise) in a $560 \mu 1$ suspension were added to the E-plate. Paclitaxel or carboplatin prepared in $40 \mu \mathrm{l}$ of culture medium was added to the E-plate cell cultures at $24 \mathrm{~h}$ after cell seeding. The CI was monitored in real time for $96 \mathrm{~h}$ from the time of cell seeding. The $\mathrm{IC}_{50}$ values were calculated using RTCA Data Analysis Software version 1.0 (ACEA Biosciences, Inc.).

\subsubsection{Combined paclitaxel/carboplatin treatment}

All treatment protocols are summarized as shown in Table 1. E-plate equilibration, setting of baseline values, and cell seeding were performed as described above for single-agent treatment. All treatment protocols were performed $24 \mathrm{~h}$ after cell seeding into E-plates. For schedule 1 treatment, paclitaxel ( $40 \mathrm{nM}$ final concentration) was added to the culture medium followed by incubation for $1 \mathrm{~h}$, and then replaced with carboplatin ( $20 \mu \mathrm{M}$ final concentration) followed by incubation for an additional $1 \mathrm{~h}$. The cells were then returned to fresh culture medium until the end of the experiment. For schedule 2 treatment (corresponding to TC therapy used for the clinical treatment of cancer patients), paclitaxel was added to the culture medium (13 nM final concentration) followed by incubation for $3 \mathrm{~h}$, and then replaced with carboplatin ( $20 \mu \mathrm{M}$ final concentration) followed by incubation for another $1 \mathrm{~h}$. The cells were then returned to a fresh culture medium and remained in this medium until the end of the experiment. For schedule 3 treatment that was performed to evaluate cytotoxicity associated with paclitaxel exposure time, paclitaxel ( $40 \mathrm{nM}$ final concentration) was added to the culture medium and the cells were then monitored for $72 \mathrm{~h}$ in the presence of the drug. Schedule 4 treatment was used as a control for schedule 3 treatment and involved the addition of paclitaxel ( $40 \mathrm{nM}$ final concentration) to the culture medium and incubation for $1 \mathrm{~h}$ before returning the cells to a fresh culture medium for the remainder of the experiment.

Table 1

Summary of combined paclitaxel and carboplatin treatment schedules based on TC therapy.

\begin{tabular}{ll}
\hline & \multicolumn{1}{c}{ Schedule during $72 \mathrm{~h}$ monitoring using RTCA system } \\
\hline Schedule 1 & $\begin{array}{l}\text { 1 h in paclitaxel }(40 \mathrm{nM}) \text { followed by } 1 \mathrm{~h} \text { in carboplatin }(20 \mu \mathrm{M}) . \\
\text { After that, } 70 \mathrm{~h} \text { in fresh medium. }\end{array}$ \\
Schedule 2 & $\begin{array}{l}\text { 3 h in paclitaxel }(13 \mathrm{nM}) \text { followed by } 1 \mathrm{~h} \text { in carboplatin }(20 \mu \mathrm{M}) . \\
\text { After that, } 68 \mathrm{~h} \text { in fresh medium. }\end{array}$ \\
Schedule 3 & $\begin{array}{l}72 \mathrm{~h} \text { in paclitaxel }(40 \mathrm{nM}) \\
\text { Schedule } 4\end{array}$ \\
\hline
\end{tabular}




\subsection{Western blotting and quantification of protein levels in HUVECs treated with paclitaxel and carboplatin}

Western blotting was performed to detect the protein expressions of intercellular adhesion molecule-1 (ICAM-1), vascular cell adhesion molecule-1 (VCAM-1), nuclear factor (NF)- $\kappa B$,

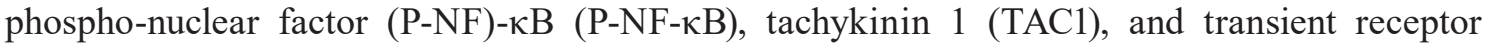
potential vanilloid 1 (TRPV1) in HUVECs $72 \mathrm{~h}$ after treatment with paclitaxel and carboplatin. Cells were lysed in a lysis buffer and the total protein concentrations of the lysates were then determined by bicinchoninic acid assay (BCA; Pierce Biotechnology, Rockford, IL, USA). Cell lysates (containing equal amounts of protein) were separated by sodium dodecyl sulfate-polyacrylamide gel electrophoresis (SDS-PAGE; Bio-Rad, Hercules, CA, USA) and protein bands were then transferred to polyvinylidene difluoride membranes (Bio-Rad). The membranes were blocked with Blocking One (Nacalai Tesque, Inc., Kyoto, Japan) overnight at $4{ }^{\circ} \mathrm{C}$ and then incubated for $1 \mathrm{~h}$ at room temperature (RT) with primary antibodies. Antibodies against ICAM-1 (ab109361; Abcam, Cambridge, UK), VCAM-1 (ab134047; Abcam), P-NF-кB; (Cell Signaling Technology, Beverly, MA), TAC1 (13839-1-AP, Proteintech Group Inc., Rosemont, USA), and TRPV1 (22686-1-AP, Proteintech Group Inc.) were used at a 1:1000 dilution in blocking solution. The antibody against glyceraldehyde 3-phosphate dehydrogenase (GAPDH) (ACR001PT; Acris Antibodies, Inc., San Diego, CA, USA) was used at a 1:10000 dilution in blocking solution. The membranes were then washed three times and subsequently incubated for $1 \mathrm{~h}$ at RT with a secondary antibody (horseradish peroxidase-conjugated speciesspecific antibody). Immunoreactive bands were visualized using ImmunoStar LD reagent (Wako Pure Chemical Industries).

\subsection{Statistical analysis}

All data are shown as the mean \pm standard deviation (SD) of three independent experiments. CI data obtained using the RTCA system were analyzed by two-way repeated analysis of variance (ANOVA) followed by Tukey's honesty significant difference (HSD) test. The statistical analysis was carried out using 13 points from $24 \mathrm{~h}$ to $96 \mathrm{~h}$ in Figs. 1-4. Western blotting data were analyzed by one-way repeated ANOVA followed by the Bonferroni test. Statistical significance was accepted at the $p<0.05$ or $p<0.01$ level.

\section{Results}

\subsection{Dose-dependent effects of single-treatment paclitaxel or carboplatin on HUVEC proliferation}

In untreated HUVEC cultures, CI values increased with time as cell proliferation within the E-plate increased, thereby inhibiting electrode impedance (Fig. 1). Paclitaxel suppressed this increase in $\mathrm{CI}$ values in a dose-dependent manner. Furthermore, where concentrations of over $6.2 \mathrm{nM}$ paclitaxel were used, this suppression in CI values lasted for up to $96 \mathrm{~h}$. As shown in Fig. 2, 


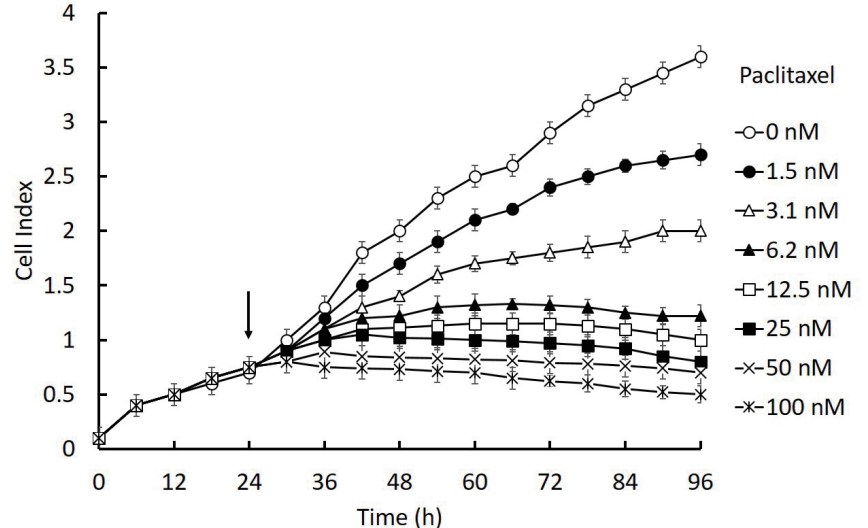

Fig. 1. Dose-dependent effects of single-treatment paclitaxel on HUVEC proliferation. Data represent means $\pm \mathrm{SD}(n=3)$. The black arrow indicates the time of addition of anticancer agents.

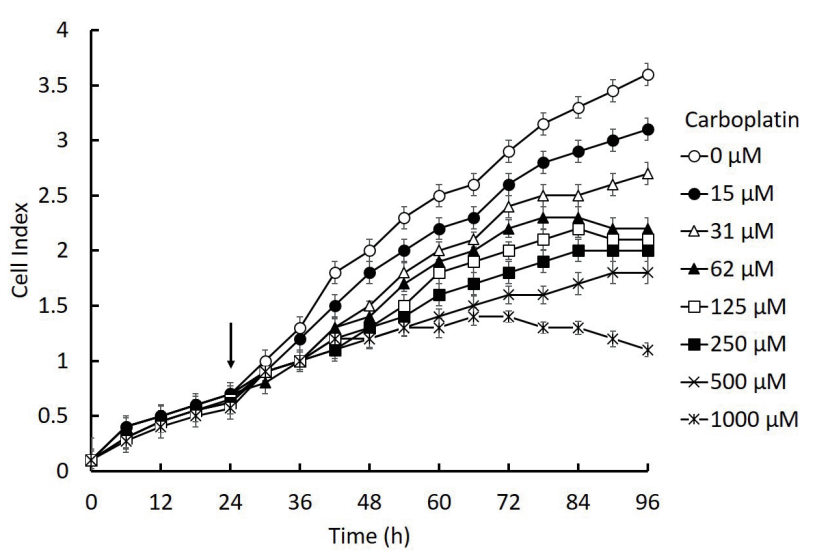

Fig. 2. Dose-dependent effects of single-treatment carboplatin on HUVEC proliferation. Data represent means $\pm \mathrm{SD}(n=3)$. The black arrow indicates the time of addition of anticancer agents.

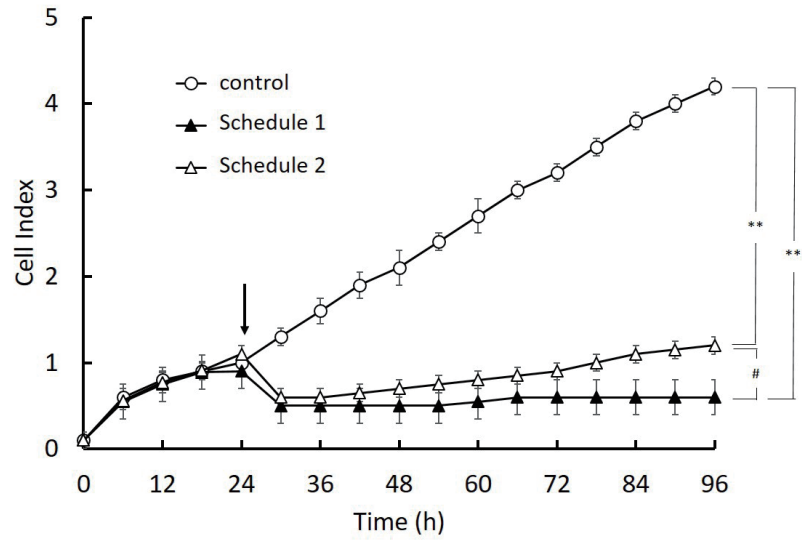

Fig. 3. Effect of combined paclitaxel and carboplatin treatment (TC therapy) on HUVEC proliferation. Data represent means $\pm \mathrm{SD}(n=3)$. The black arrow indicates the time of addition of anticancer agents. ${ }^{* *} p<0.01$ versus control, ${ }^{*} p<0.05$ versus schedule 1 (Tukey's HSD test /ANOVA).

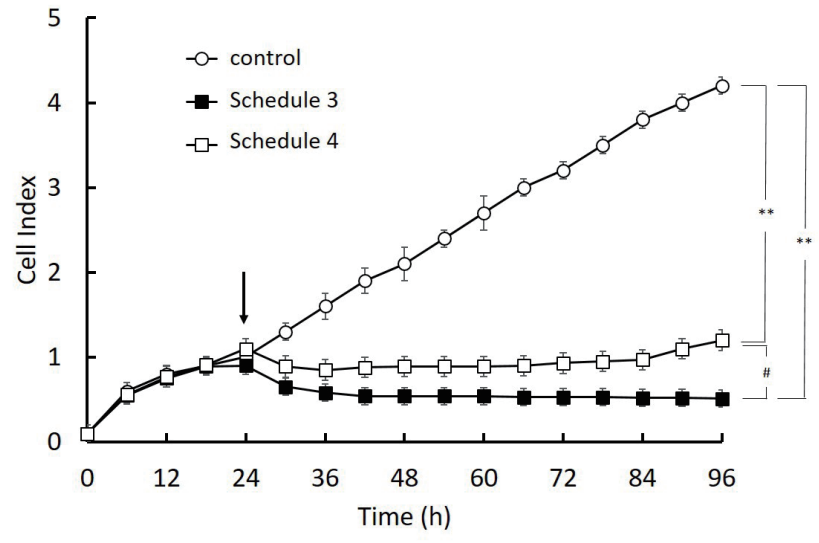

Fig. 4. Effect of exposure time of paclitaxel on HUVEC proliferation. Data represent means \pm SD $(n=3)$. The black arrow indicates the time of addition of anticancer agents. ${ }^{* *} p<0.01$ versus control, ${ }^{\#} p<0.05$ versus schedule 3 (Tukey's HSD test /ANOVA).

carboplatin also suppressed the increase in CI values in HUVEC cultures in a dose-dependent manner, and the CI profile was similar to that found for paclitaxel. However, CI values for HUVEC cultures treated with carboplatin within the 15-500 $\mu \mathrm{M}$ range increased gradually with time, demonstrating lower profile slopes than that of the control. In cultures treated with $1000 \mu \mathrm{M}$ carboplatin, the increase in the CI profile was suppressed. These results suggest that paclitaxel is more cytotoxic towards HUVECs than carboplatin. 


\subsection{Effects of combined paclitaxel and carboplatin treatment (TC therapy) on HUVEC proliferation}

In clinical settings, TC combination therapy involves the intravenous administration of a $13 \mathrm{nM}$ paclitaxel solution over a $3 \mathrm{~h}$ period and a $20 \mu \mathrm{M}$ carboplatin solution over a $1 \mathrm{~h}$ period. Considering the findings from our in vitro studies (Figs. 1 and 2), it is possible that, in clinical settings, the concentration of paclitaxel at the time of injection has cytotoxic consequences, including angialgia, for the cells of the human vascular endothelium. This is because these cells are exposed to a high local concentration of paclitaxel. In this study, we designed two experimental schedules using HUVECs as a model of the vascular endothelium to elucidate the effects of "exposure time" and "concentration" on angialgia, two important factors in the clinical dosing schedule.

As shown in Fig. 3, the CI values for schedule 2 were higher than those for schedule 1 over the course of the experimental period (schedule details are described in Materials and Methods). These results suggest that exposure to a low but sustained concentration of paclitaxel (schedule 2) is less cytotoxic for endothelial cells than a short exposure to a higher concentration of the drug. The CI values were higher for schedule 4 than for schedule 3 over the course of the experimental period (Fig. 4). These results suggest that, when the concentration of the anticancer reagent is constantly high, the cytotoxicity of paclitaxel towards endothelial cells is dependent on the exposure time. Taken together, our findings show that it is important to administer less than $13 \mathrm{nM}$ paclitaxel and to administer it slowly in order to reduce cytotoxicity towards the cells of the vascular endothelium.

\subsection{Effects of paclitaxel and carboplatin on the expressions of adhesion, inflammation, and pain-related markers}

Changes in the protein levels of ICAM-1, VCAM-1, P-NF- $\mathrm{B} / \mathrm{NF}-\kappa \mathrm{B}, \mathrm{TAC} 1$, and TRPV1 in HUVECs exposed to three different concentrations of paclitaxel and carboplatin were determined by western blotting. The concentrations chosen for each drug included their clinical concentrations ( $40 \mathrm{nM}$ for paclitaxel, $20 \mu \mathrm{M}$ for carboplatin), and five and ten times the clinical concentrations. As shown in Fig. 5, paclitaxel treatment resulted in a dose-dependent increase in the expression levels of ICAM-1 and VCAM-1. Conversely, carboplatin had no effect on the expression of VCAM-1.

Furthermore, while carboplatin elevated ICAM-1 levels, this effect was not dose-dependent. As shown in Fig. 6, the phosphorylation of NF- $\kappa B$ was enhanced by paclitaxel treatment, but was unaffected by carboplatin treatment. Figure 7 shows that the expression of TAC1, a receptor for substance $\mathrm{P}$, was suppressed by paclitaxel treatment but unaffected by carboplatin treatment. This phenomenon was also observed for TRPV1, a cation channel involved in pain signaling. Furthermore, NF- $\kappa \mathrm{B}$ phosphorylation was enhanced under the schedule 1 treatment setting but not under the schedule 2 treatment setting (Fig. 8). These data suggest that schedule 2 is more preferable than schedule 1 for reducing angialgia/phlebitis during chemotherapy

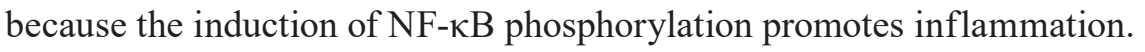



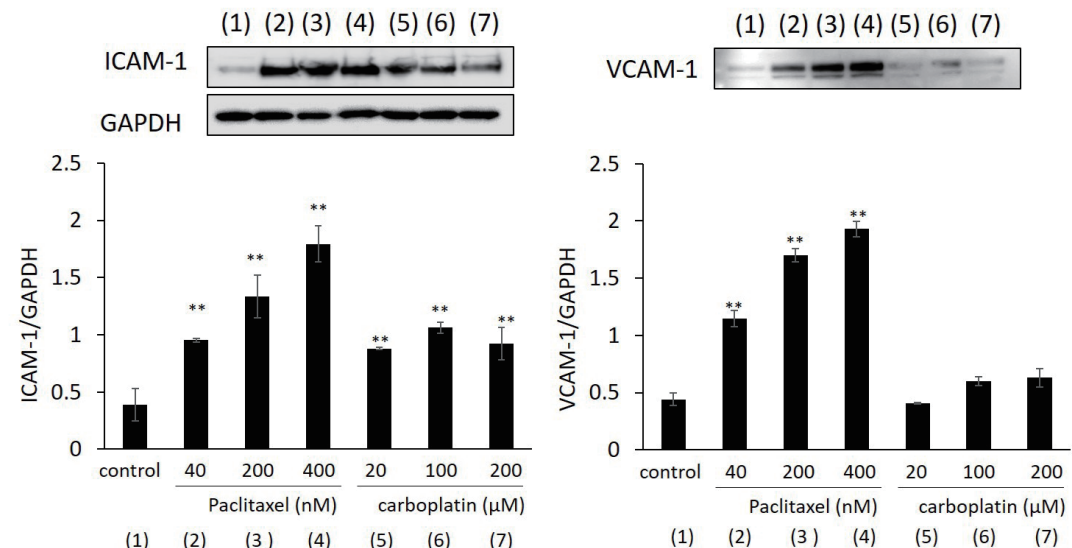

Fig. 5. ICAM-1 and VCAM-1 levels in HUVECs at $72 \mathrm{~h}$ after incubation with paclitaxel or carboplatin. Data represent means $\pm \mathrm{SD}(n=3) .{ }^{* *} p<0.01$ versus control (Bonferroni test/ANOVA).

(1) (2) (3) (4) (5) (6) (7)

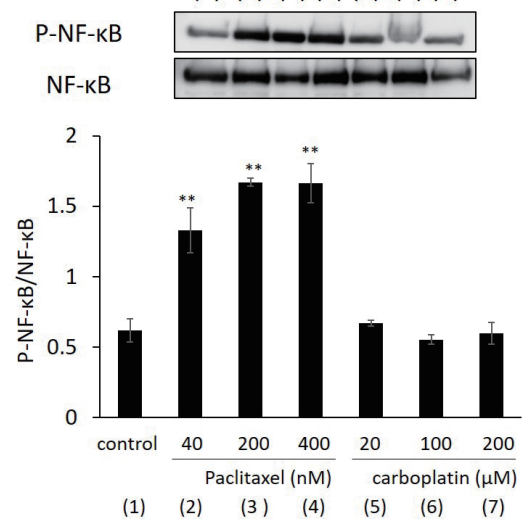

Fig. 6. NF- $\mathrm{BB}$ phosphorylation levels in HUVECs at $72 \mathrm{~h}$ after treatment with paclitaxel or carboplatin. Data represent means $\pm \mathrm{SD}(n=3) .{ }^{* *} p<0.01$ versus control (Bonferroni test/ANOVA).

(1) (2) (3) (4) (5) (6) (7)

TAC1

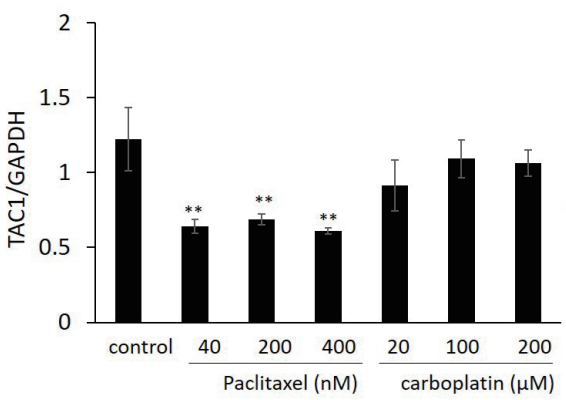

$\begin{array}{lllllll}\text { (1) } & \text { (2) } & \text { (3) } & \text { (4) } & \text { (5) } & \text { (6) } & \text { (7) }\end{array}$
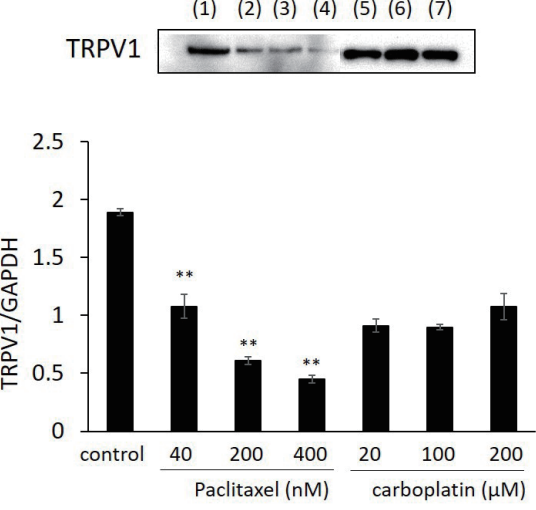

$\begin{array}{llllll}\text { (1) } & \text { (2) } & \text { (3) } & \text { (4) } & \text { (5) } & \text { (6) }\end{array}$

Fig. 7. TAC1 and TRPV1 levels in HUVECs at $72 \mathrm{~h}$ after treatment with paclitaxel or carboplatin. Data represent means $\pm \mathrm{SD}(n=3) .{ }^{* *} p<0.01$ versus control (Bonferroni test/ANOVA). 


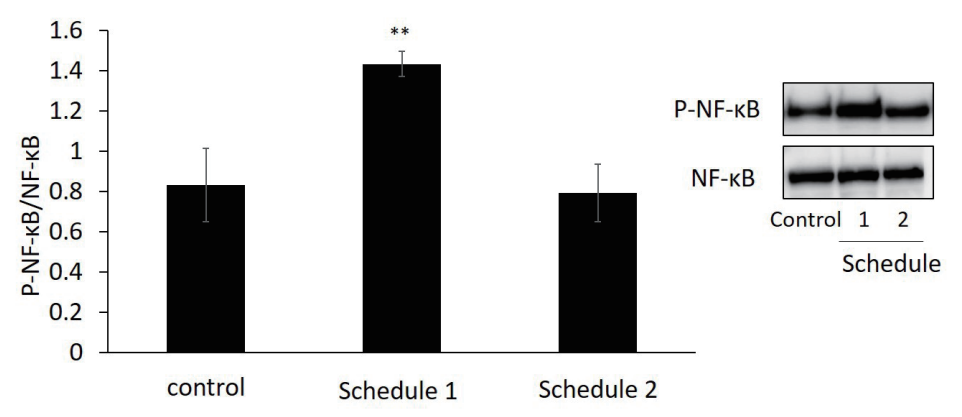

Fig. 8. NF- $\kappa \mathrm{B}$ phosphorylation levels in HUVECs at $72 \mathrm{~h}$ after TC therapy as indicated in schedule 1 and 2 protocols. Data represent means $\pm \mathrm{SD}(n=3) .{ }^{* *} p<0.01$ versus control (Bonferroni test/ANOVA).

\section{Discussion}

As shown in Figs. 1 and 2, paclitaxel was more cytotoxic towards HUVECs than carboplatin because there is a positive correlation between the CI value and cytotoxicity. These findings are in agreement with previous data. ${ }^{(9)}$ The results presented in Figs. 3 and 4 suggest that inflammation and angialgia can be avoided by using a low concentration of the drug during chemotherapy. This is because these data suggest that cytotoxicity and angialgia may be induced when CI values are suppressed, such as during schedule 1 and 3 treatments.

This study was the first trial to establish a new evaluation method for the prediction of angialgia/phlebitis using RTCA system, so we attempted to implement it with a simpler experimental schedule such as schedules 1,2 , and 3 in order to make it easier to compare results. The drug solution concentration in the schedule was set as that to be administered and the solution was assumed to be not diluted. In general, pharmaco-kinetics is evaluated using the area under concentration (AUC) to determine the drug efficacy and systemic side effects of anticancer reagents because anticancer reagents because diluted in the blood circulation after intravenous administration. However, in the case of angialgia/phlebitis as the side effect of the anticancer reagents, the pain occurs in the upper limbs near the indwelling needle (in local) at the time of administration and reduces the patient's QOL. For the above reasons, the important point in this evaluation system for predicting angialgia/phlebitis using HUVECs is to observe the intravascular cell reaction as CI value in real time under high-concentration exposure condition immediately after administration in local, rather than the effect of the diluted drug solution after administration. Although only four schedules based on fixed-concentration TC therapy were examined in this study, the observation that cell proliferation did not recover immediately after washing out, suggests that cell damage due to temporarily high concentration exposure continues and suppresses subsequent cell proliferation.

The RTCA system detects impedance, and this is influenced by the proximity of cells to the electrodes. While the system can therefore detect changes in cell number, the strength of attachment, or morphology, it cannot directly evaluate pain or inflammation. For this reason, in this study, we combined protein expression analysis with the RTCA system to determine whether this combined approach could be used to detect the risk of developing angialgia/ 
phlebitis. The results shown in Figs. 5 and 6 suggest that a decrease in CI values may indicate an increased degree of inflammation. It has been reported that ICAM-1 and VCAM-1 levels significantly increase in tumor necrosis factor (TNF)- $\alpha$-stimulated cells. ${ }^{(10)}$ Furthermore, it has been reported that ICAM-1 and VCAM-1 levels significantly increase in response to NF- $\kappa \mathrm{B}$ signaling during inflammation induced by lipopolysaccharides. ${ }^{(11)}$ Given the findings of these previous studies, our data suggest that paclitaxel induces a stronger inflammatory response than carboplatin.

In clinical settings, cell toxicity, irritation, and angialgia are more commonly associated with paclitaxel than with carboplatin. Our protein expression data suggest that NF- $\mathrm{B}$ phosphorylation is an important surrogate for pain considering that $40 \mathrm{nM}$ paclitaxel is commonly associated with angialgia, while $20 \mu \mathrm{M}$ carboplatin is not. In addition, in Fig. 8, our data suggest that schedule 2 treatment would be less likely to be associated with pain than schedule 1 treatment. This is because the significant decrease in CI values and the significant increase in NF- $\mathrm{BB}$ phosphorylation may predict the appearance of pain.

The expression levels of TAC1 and TRPV1, a pain-associated receptor and an ion channel, respectively, decreased following paclitaxel treatment, as shown in Fig. 7. Previous studies have shown that inhibition of TRPV1 prevents chemotherapy-induced peripheral neuropathic pain in vivo. ${ }^{(12-14)}$ It has also been reported that high-concentration paclitaxel $(300 \mathrm{nM})$ suppresses the release of the calcitonin gene-related peptide (CGRP) and alters the function of TRP channel activity in sensory neurons in vitro. ${ }^{(15,16)}$ In these previous studies, the observed reduction in the expression levels of TAC1 and TRPV1 was attributed to the high concentrations of paclitaxel used (40-400 nM).

A large number of studies using rodent dorsal root ganglion neurons or in vivo rodent models have been performed to elucidate the mechanisms of angialgia, such as those underlying paclitaxel-induced neuropathic pain. ${ }^{(17,18)}$ However, these mechanisms are likely complex, and a quantitative evaluation of angialgia that represents the degree of pain caused by chemotherapy is difficult using conventional methods. Human evaluation of pain is confounded by the fact that it is a subjective phenomenon. The pain response is a phenotype elicited via the central nerve system. The peripheral nerve system also mediates pain responses that involve normal cell injury, such as the exposure of the vascular endothelium to anticancer drugs during chemotherapy. Given this, our RTCA method may be useful for the prediction of angialgia as a side effect of cell cytotoxicity because the system can be used to observe the response of the peripheral vascular endothelium and predict or estimate cytotoxicity through real-time monitoring using CI values from the RTCA system, although the principle of the RTCA system is not direct cytotoxicity evaluation. Furthermore, by combining the RTCA system with the analysis of NF- $\mathrm{KB}$ phosphorylation, such an approach may be able to evaluate the degree of pain induced by a given treatment regimen.

A major advantage of using the RTCA system is that treatments added to the wells of the E-plate can be washed out during the course of sample measurement. This means that vascular endothelial cell responses can be assessed dynamically in an environment where the levels of cytotoxic agents change with time, similarly to chemotherapy where anticancer reagents are changed according to the dosing schedule. This method may therefore be considered to be the first in vitro clinical pain evaluation system that is in line with clinical practice. 


\section{Conclusion}

Our results demonstrated that the method using the RTCA system is useful for the detection of the risk of developing angialgia/phlebitis in HUVECs treated with paclitaxel, when the system is combined with an analysis of NF-kB phosphorylation. Evaluation methods that offer alternatives to those that are particularly difficult to perform in humans are very useful. Additionally, in terms of real-time evaluation during the administration of anticancer reagents in chemotherapy, we are unaware of other methods that can provide a more superior evaluation because the RTCA system can evaluate cell reaction profiles both automatically and in realtime.

\section{Acknowledgements}

This work was supported in part by the Japan Society for the Promotion of Science (JSPS) KAKENHI Grant Number JP17K08477 and Fukuoka Foundation for Sound Health Cancer Research Fund.

\section{References}

1 J. Z. Xing, L. Zhu, J.A. Jackson, S. Gabos, X. J. Sun, X. B. Wang, and X. Xu: Chem. Res. Toxicol. 18 (2005) 154. https://doi.org/10.1021/tx049721s

2 J. Z. Xing, L. J. Zhu, S. Gabos, and L. Xie: Toxicol. In Vitro. 20 (2006) 995. https://doi.org/10.1016/ j.tiv. 2005.12 .008

3 J. Zhu, X. Wang, X. Xu, and Y. A. Abassi: J. Immunol. Methods 309 (2006) 25. https://doi.org/10.1016/ j.jim.2005.10.018

4 B. Xi, N. Yu, X. Wang, X. Xu, and Y. A. Abassi: Biotechnol. J. 3 (2008) 484. https://doi.org/10.1002/ biot. 200800020

5 M. Morioka, M. Hazekawa, T. Kawakubo-Yasukochi, T. Nishinakagawa, S. Nakamura, and M. Nakashima: Pharmacol. Pharm. 7 (2016) 255. https://doi.org/10.4236/pp.2016.77032

6 M. Hazekawa, M. Morioka, T. Nishinakagawa, T. Kawakubo-Yasukochi, S. Nakamura, and M. Nakashima: Electron. J. Biol. 13 (2017) 56. http://ejbio.imedpub.com/assessment-of-cytotoxicity-of-imatinib-for-oralsquamous-cellcarcinoma-by-a-realtime-cell-analysis-system.php?aid=18089

7 M. Hazekawa, T. Nishinakagawa, T. Kawakubo-Yasukochi, and M. Nakashima: Exp. Ther. Med. 18 (2019) 3197. https://doi.org/10.3892/etm.2019.7876

8 A. Abed, M. J. Khoshnoud, M. Taghian, M. Aliasgharzadeh, and A. Mesdaghinia: Iran J. Basic. Med. Sci. 20 (2017) 1182. https://doi.org/10.22038/IJBMS.2017.9500

9 J. M. Quinn, M. M. Greenwade, M. L. Palisoul, G. Opara, K. Massad, L. Guo, P. Zhao, H. Beck-Noia, I. S. Hagemann, A. R. Hagemann, C. K. McCourt, P. H. Thaker, M. A. Powell, D. G. Mutch, and K. C. Fuh: Mol. Can. Ther. 18 (2019) 389. https://doi.org/10.1158/1535-7163.MCT-18-0537

10 A. Das, V. Sudhahar, M. Ushio-Fukai, and T. Fukai: Am. J. Physiol. Cell Physiol. 317 (2019) C1161-C1171. https://doi.org/10.1152/ajpcell.00264.2019

11 J. Sun, N. Huang, W. Ma, H. Zhou, and K. Lai: Mol. Med. Rep. 19 (2019) 1817. https://doi.org/10.3892/ mmr.2019.9807

12 Y. Wu, J. Chen, and R. Wang: Neuroreport 30 (2019) 288. https://doi.org/10.1097/WNR.0000000000001199

13 J. Luo, A. Bavencoffe, P. Yang, J. Feng, S. Yin, A. Qian, W. Yu, S. Liu, X. Gong, T. Cai, E. T. Walters, C. W. Dessauer, and H. Hu: J. Neurosci. 38 (2018) 474. https://doi.org/10.1523/JNEUROSCI.1816-17.2017

14 Y. Li, P. Adamek, H. Zhang, C. E. Tatsui, L. D. Rhines, P. Mrozkova, Q. Li, A. K. Kosturakis, R. M. Cassidy, D. S. Harrison, J. P. Cata, K. Sapire, H. Zhang, R. M. Kennamer-Chapman, A. B. Jawad, A. Ghetti, J. Yan, J. Palecek, and P. M. Dougherty: J. Neurosci. 35 (2015) 13487. https://doi.org/10.1523/JNEUROSCI.1956-15.2015

15 L. M. Darby, H. Meng, and J. C. Fehrenbacher: Mol. Cell. Neurosci. 82 (2017) 105. https://doi.org/10.1016/ j.men.2017.04.001 
16 S. K. Pittman, N. G. Gracias, M. R. Vasko, and J. C. Fehrenbacher: Exp. Neurol. 253 (2014) 146. https://doi. org/10.1016/j.expneurol.2013.12.011

17 N. P. Staff, J. C. Fehrenbacher, M. Caillaud, M. I. Damaj, R.A. Segal, and S. Rieger: Exp. Neurol. 324 (2020) 113121. https://doi.org/10.1016/j.expneurol.2019.113121

18 P. N. Huynh, D. Giuvelis, S. Christensen, K. L. Tucker, and J. M. McIntosh: Mar. Drugs. 18 (2020) 12. https:// doi.org/10.3390/md18010012 